\title{
Polynomisches Kontaktmodell zur Abbildung pastösen Materialverhaltens in DEM-Simulationen
}

\author{
Felix Platzer $^{1}$ und Eric Fimbinger ${ }^{2}$
}

${ }^{1}$ Lehrstuhl für Bergbaukunde - Fördertechnik und Konstruktionslehre, Montanuniversität Leoben, Leoben, Österreich

${ }^{2}$ Lehrstuhl für Aufbereitung und Veredlung, Montanuniversität Leoben, Leoben, Österreich

Eingegangen 21. Dezember 2021; angenommen 6. Januar 2022; online publiziert 7. Februar 2022

\begin{abstract}
Zusammenfassung: Zur Simulation pastöser Materialien in der DEM wird ein Kontaktmodell vorgestellt, welches beim Kontaktverlust zweiter ursprünglich in Kontakt befindlicher Partikel (diskrete Elemente) eine anziehende Kraft (Kohäsionskraft) basierend auf einem polynomischen Ansatz bildet. Dieses folglich kapillare Kontaktmodell ist somit in der Lage, pastöse Materialien für Industrie orientierte DEM-Simulationen nachzubilden.

Zum Abgleich der hierbei modellierten Materialien wird der Slump-Test als standardisierte und weit verbreitete Methode vorgestellt, wobei über Parametervariation ein Abgleich des im Labor festgestellten Verhaltens des pastösen Materials mit jenem Verhalten des digitalen Modells, aufgebaut als Partikelansammlung in der DEM-Simulation, durchgeführt wird.
\end{abstract}

Schlüsselwörter: DEM, Pasten, Pastöses Material, Kapillare Kohäsion, Haldenmaterial, PolynomKontaktmodell

Polynomial Contact Model for Depicting Pasty Material Behaviour in DEM Simulations

Abstract: For the simulation of pasty materials in the DEM, a contact model is presented, which forms an attractive force (cohesive force) based on a polynomial function in the case of contact loss of particles originally in contact with each other (discrete elements). Consequently, this capillary contact model is thus able to reproduce pasty materials for industry-oriented DEM simulations.

For the calibration of the modelled materials, the slump test is presented as a standardised and widely used method,

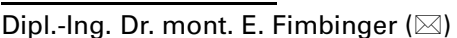

Lehrstuhl für Aufbereitung und Veredlung,

Montanuniversität Leoben,

Franz Josef-Straße 18,

8700 Leoben, Österreich

eric.fimbinger@unileoben.ac.at whereby a comparison of the behaviour of the pasty material determined in the laboratory with that of the digital model set up as large amount of particles in the DEM simulation is carried out via parameter variation.

Keywords: DEM, Pastes, Pasty material, Capillary cohesion, Tailings, Polynomial contact model

\section{Pastöses Material}

Gemische eines feinkörnigen Feststoffes mit einer Flüssigkeit, wobei der Feststoff einen größeren Teil des Gemischs ausmacht, werden als pastöse Materialien bezeichnet. Bei vielen technischen Prozessen, wie z. B. der Gewinnung von diversen Metallen ( $\mathrm{Cu} \mathrm{Ag} \mathrm{Au}$ ), wird mit großen Mengen an pastösem Material gearbeitet. Je höher der Wasseranteil in dem Gemisch ist, desto flüssig-ähnlicher ist das Materialverhalten.

Beispiele solch pastöser Materialien finden sich in der Betonverarbeitung, wie in Abb. 1 beispielhaft gezeigt, als Schlämme in der Haldentechnik, in der Sprengtechnik beim Einsatz pumpfähiger Sprengstoffe sowie auch in vielen weiteren Industriefeldern, beispielsweise der Kunststoff(-Spritzguss)-verarbeitung, in der Tiefbohrtechnik und auch der Nahrungsmittel- oder Agrarindustrie.

Das für pastöse Materialien charakteristische, hochviskose und streichfähige Verhalten beruht auf anziehenden Kräften zwischen einzelnen Partikeln, welche auf dem Kapillareffekt basieren. Der Kapillareffekt innerhalb granularer Medien beschreibt das Verhalten von sogenannten Kapillarbrücken, welche zwischen Partikeln des Mediums bei der Zugabe einer Flüssigkeit gebildet werden. Aufgrund der Oberflächenspannung der Flüssigkeit und dem Kapillaren Druck, der Druckdifferenz zwischen der Umgebenden Luft und dem Flüssigkeitsdruck, auch als Laplace Druck bekannt, wirken dadurch anziehende Kräfte zwischen den Partikeln $[2,3]$. Mit größer werdender Entfernung zwischen den Partikeln nimmt diese Kapillarkraft immer weiter ab, bis sie 


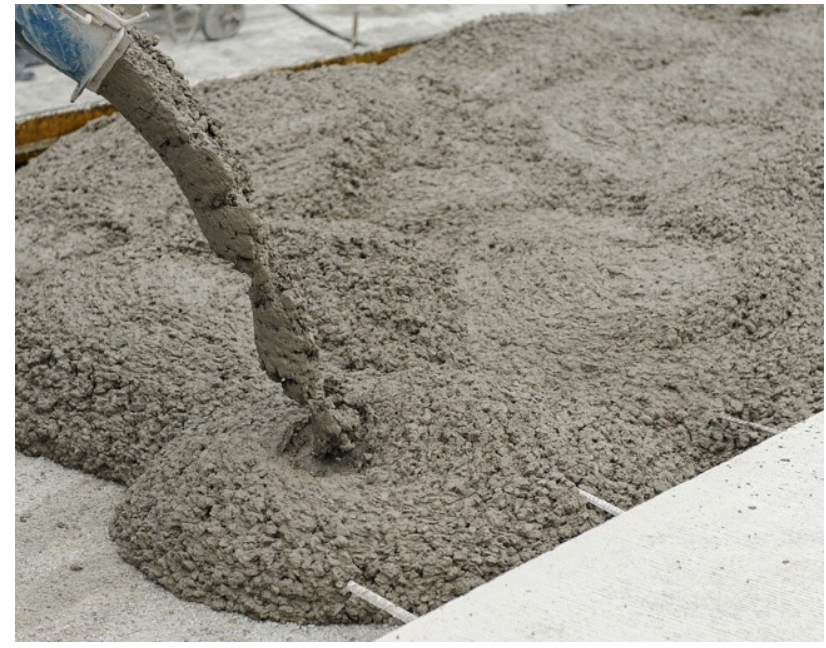

Abb. 1: Beton als Beispiel eines Materials mit typischem pastösen Verhalten [1]

ab einem bestimmten Abstand reißt, was zu einem abrupten Abfall der Kraft auf 0 führt [4]. Je höher der Wassergehalt ist, desto mehr Kapillarbrücken wachsen zu Clustern zusammen, die irgendwann alle Poren ausfüllen. Ist dieser Zustand erreicht, so führt die zusätzliche Zugabe von weiterem Wasser zu einer schnellen Abnahme der Kapillarkraft [4]. Sobald der Flüssigkeitsdruck durch weitere Wasserzugabe auf den umgebenden Luftdruck steigt, wird ein Zustand erreicht, bei dem das Material stark an kapillarer Kohäsion einbüßt.

\subsection{Numerische Simulation pastöser Materialien}

Das Materialverhalten von granularen Medien kann in einer Diskreten Elemente (DEM) Simulation abgebildet werden. Die Kalibrierung der Simulationsparameter erfolgt dabei durch den Vergleich von Laborversuchen, welche ein charakteristisches Materialverhalten wiederspiegeln, mit dem Ergebnis von Kalibrierungssimulationen.

Neben DEM-Simulationen weisen auch Smoothed Particle Hydrodynamic (SPH) Simulationen vielversprechende Möglichkeiten für die Nachbildung von pastösen Materialien auf. Bei SPH Simulationen wird der Fluss eines Kontinuum-Mediums durch diskrete Elemente abgebildet. Im Vergleich zur DEM wird nicht nur den Einfluss der direkten Nachbarn eines Elements, sondern auch jener von weiter entfernten Elementen mit abnehmender Wirkung berück- sichtigt [5]. Obwohl diese Simulationsmethode meist zur Abbildung reiner Fluide verwendet wird, kann eine Kalibration durchgeführt werden, um hochviskose Aufschlämmungen zu replizieren. Da jedoch selbst sehr hochviskose Flüssigkeiten selbst nach sehr langer Zeit nicht aufhören zu fließen, ist diese Methode nur für die Simulation von pastösen Medien mit einem sehr hohen Wassergehalt geeignet und werden im Laufe des Projektes nicht näher betrachtet.

In diesem Projekt wurden mehrere DEM-Kontaktmodelle, welche anziehenden Kräfte zwischen Partikeln in Abhängigkeit des Abstands der Partikel beschreiben, analysiert und anhand ihrer Fähigkeit beurteilt, pastöses Materialverhalten mit hohem Wassergehalt darzustellen, indem die Simulationsergebnisse mit einem Slump-Test verglichen werden. Ein äußerst vielversprechendes Kontaktmodell wurde anschließend optimiert, um eine minimale Rechenzeit von technischen Prozessen zu ermöglichen.

Viele bereits existierende Kontaktmodelle, wie Kohäsionsmodelle, berücksichtigen zwar auch anziehende Kräfte zwischen Partikeln, beruhen jedoch auf der Anziehung gleicher Stoffe, was bei pastösen Materialien jedoch nicht der Fall ist. Aus diesem Grund wurden nur Kontaktmodelle evaluiert, welche auf dem Kapillareffekt basieren.

Die Kapillarkräfte in Abhängigkeit des Abstandes zwischen den Partikel, zwischen denen sich die Kapillarbrücken- bzw. Cluster gebildet haben, weisen typischerweise einen exponentiell abfallenden Verlauf auf, bis sie ab einer bestimmten Distanz zwischen den Partikeln abrupt auf 0 abfallen. Dieser charakteristische Weg-KraftZusammenhang wurde als Basis für die Entwicklung des Kontaktmodells hergezogen.

Weitere Details zu der im Folgenden vorgestellten Simulations- bzw. Modellierungsmethode pastöser Materialien wurden bereits in [6] gezeigt.

\section{DEM-Kontaktmodell}

Die Partikel, welche für die Simulation von pastösem Material verwendet werden, können als Kugeln mit einem physischen Radius und einem Kollisionsoffset, welcher kombiniert den Kontaktradius ergeben (ersichtlich in Abb. 2) angenommen werden. Bei der Überschneidung der Partikel wird aus diesem Grund zwischen einer negativen Überlappung, einer Überschneidung der Kontakt-Radien, nicht jedoch der physikalischen Radien, und einer positiven Überlappung, einer Überschneidung der physikalischen Radien, unterschieden.
Abb. 2: Schematische Beschreibung der Partikelstruktur in der DEM-Software, welche die Detektion einer negativen Überlappung (a) und eine positive Überlappung (b) veranschaulicht [6]. (Rp Physikalischer Radius, Rc Kontakt Radius)

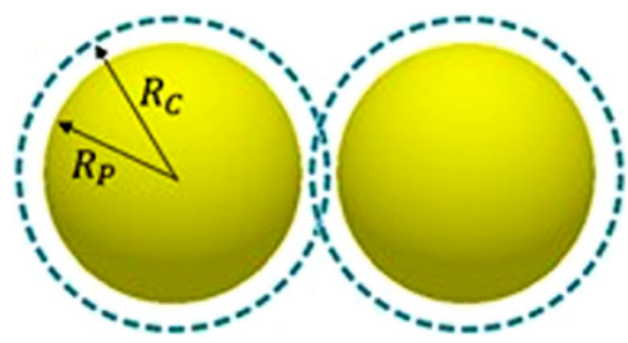

a

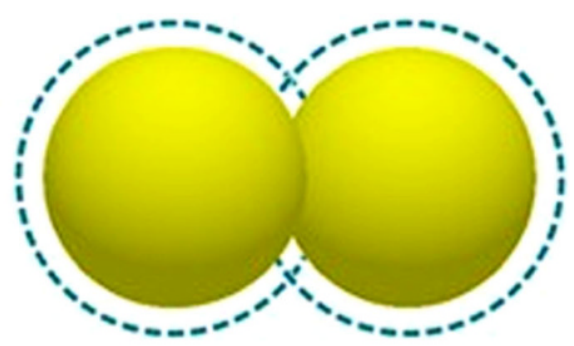

b 
Die Berechnung der Kontaktkräfte zwischen interagierenden Partikeln erfolgt in diesen zwei Interaktionsfällen wie folgend.

\subsection{Kräfte bei negativer Überlappung}

Bei der Detektion einer negativen Überlappung zwischen interagierenden Partikeln wird die Kontaktkraft anhand eines Kontaktmodells berechnet, welches den Kapillareffekt beschreibt. Der Kollisionsoffset repräsentiert in diesem Fall die Wassermenge an der Oberfläche eines Partikels, welche bei Kontakt mit einem anderen oder mehrerer Partikel eine Kapillarbrücke bzw. einen Cluster ausbildet.

Das Kontaktmodell, mit dem die Kapillarkraft zwischen den Partikeln berechnet wird, folgt einer möglichst einfachen mathematischen Funktion, um bei der Kalibrierung der Simulationsparameter günstigen Einfluss zu nehmen bzw. diesen Prozess schlussendlich zu beschleunigen. Dementsprechend ist anzumerken, dass das Kontaktmodell einer Idealisierung eines physikalisch korrekten Kraftverlaufs entspricht, um ein möglichst realistisches Gesamtverhalten des pastösen Materials mit einer möglichst einfachen Kalibrierung zu kombinieren.

Durch das Kalibrieren der Simulationsparameter in der Kalibrierungssimulation hat sich eine Polynomfunktion vor anderen einfachen mathematischen Funktionen, wie einer linearen oder exponentiellen Funktion, ersichtlich in Abb. 3, hervorgehoben, da diese nicht nur den Verlauf einer Kapillarbrücke ähnelt, sondern auch die Simulation mit einem möglichst großen Zeitschritt ermöglicht.

Die Kapillarkraft ergibt sich nach

$$
F_{\text {Kapillar }}=a \times d^{2}+b \times d+c
$$

mit $0 \geq \mathrm{d} \geq d_{c}$, wobei $d$ der Überlappung zwischen interagierenden Teilchen, $d_{c}$ jener Distanz, ab der die Kapillarbrücke reißt, und a, b und c Koeffizienten angibt, die für die Kraft-

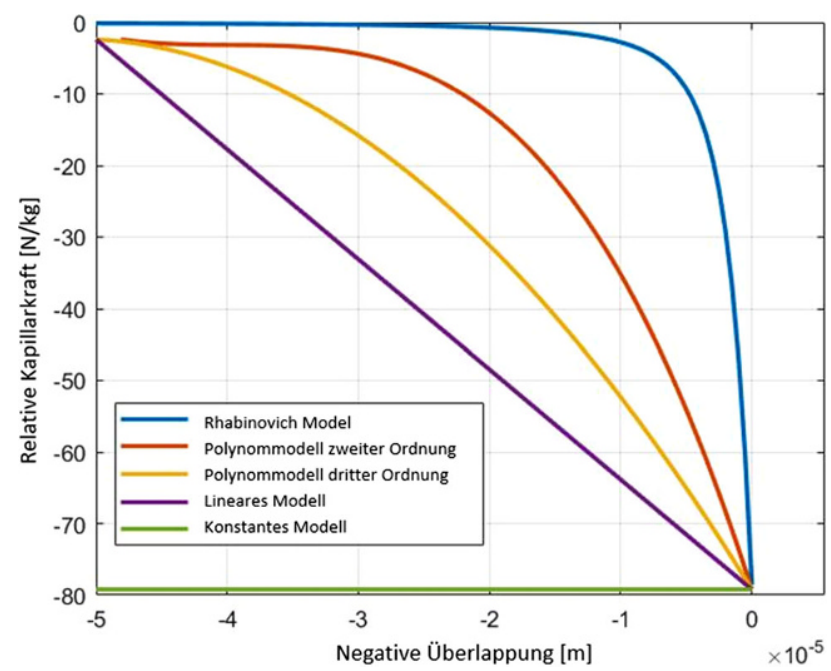

Abb. 3: Evaluierte Kraftverläufe unterschiedlicher mathematischer Modelle und eines Kapillarbrücken-Modells [7], in Abhängigkeit der negativen Überlappungen [6]
Weg-Kennlinie verantwortlich sind, welche weiters mittels Laborversuchen kalibriert werden.

\subsection{Kräfte bei positiver Überlappung}

Wird eine positive Überlappung detektiert, erfolgt die Berechnung der Kontaktkraft zwischen interagierenden Partikeln anhand des Hertz-Mindlin-Kontaktmodells, eines in der DEM typischen Kontaktmodells. Normale und tangentiale Rückfederungskräfte werden in Abhängigkeit von der Überlappung berechnet und Dämpfungskräfte in Abhängigkeit der Relativbewegung der Partikel.

Die Rückfederungskraft des Hertz-Mindlin-Kontaktmodells führt dazu, dass Partikel im Falle einer Kollision mit einem Objekt stark zurückgestoßen werden, was wiederum nicht dem Verhalten eines pastösen Materials entspricht. Um diesem Verhalten in der Simulation entgegenzuwirken, wird das Hertz-Mindlin-Kontaktmodell mit einem zusätzlichen Kontaktmodell überlagert, welches der Rückfederungskraft in Normalrichtung entgegenwirkt (ersichtlich in Abb. 4).

\section{Kalibration}

Um die Simulationsparameter für die Partikelinteraktion zu kalibrieren, werden Kalibrierungssimulationen durchgeführt, in denen ein Laborversuch nachgebildet wird. Dieser Laborversuch muss das für das zu kalibrierende Material charakteristische Verhalten in der Simulation wiedergeben. Da bereits ein standardisierter Test, ein sogenannter Slump-Test, existiert, der typischerweise für in-situ Einsätze zur Bestimmung der Zähflüssigkeit von Beton Anwendung findet (was wiederum das Mischverhältnis von Zement und Wasser widerspiegelt), wird ein leicht abgewandelter Versuch zur Simulationskalibration pastösen Materials herangezogen. Obwohl der Slump-Test ursprünglich

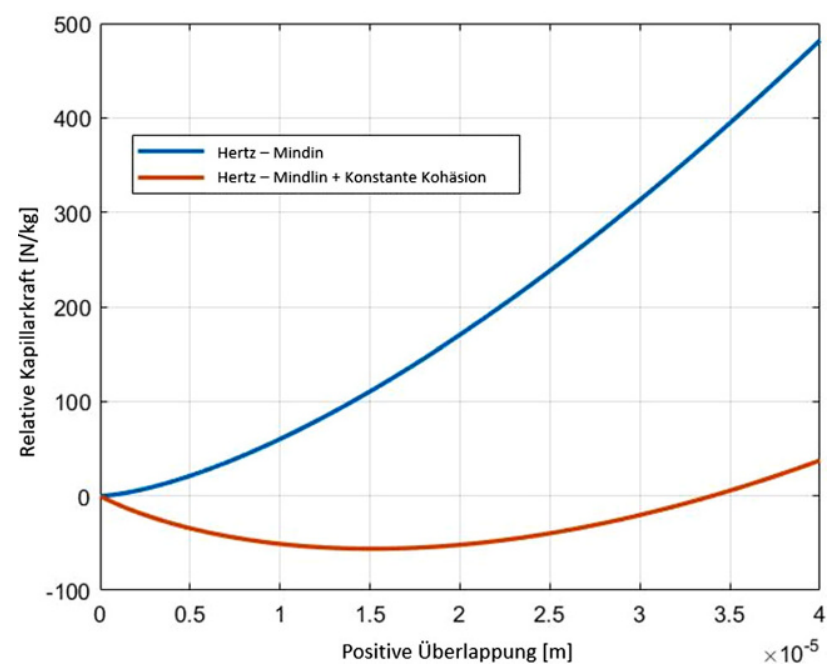

Abb. 4: Berechnete Kräfte bei positiven Überlappungen anhand des Hertz-Mindlin bzw. des Hertz-Mindlin-Modells in Kombination mit einem konstanten Kohäsionsmodell [6] 
im Tiefbau verwendet und auch bereits mehrfach mittels der DEM simuliert wurde (bspw [8]), wird er in diesem Projekt als relativ einfacher und schneller Test für das Studium an Kontaktmodellen eingesetzt, mit der Absicht, dieses vorhin angesprochene Kontaktmodell in speziellen Industrieorientierten Simulationen, wie im Bereich der Fördertechnik, anzuwenden. Der Versuch wird hierfür in zwei Schritte unterteilt, welche in Abb. 5 ersichtlich sind.

Im ersten Schritt des Versuchs, der Abb. 5a dargestellt ist, wird der kegelstumpfförmige Behälter mit konstanter Geschwindigkeit, in Abb. 5a als v ersichtlich, von der Grundplatte nach dem Befüllen mit dem pastösen Material angehoben. (Sowohl der Behälter als auch die Basisplatten sind nach DIN 12350-5 genormt.) Nachdem das Material nach dem Ausfließvorgang zu Ruhe gekommen ist, wird die verbliebene Höhe des liegenden Materials („Slump“), in Abb. 5a als h bemaßt, gemessen. Die Ausbreitungsfront des pastösen Materials fällt aufgrund der aufskalierten Partikelgröße in der Simulation nicht so flach aus wie in der Realität (siehe folgend beschriebene Abbildungen), weswegen nicht das Ausbreitungsmaß, wie in der Norm, sondern die verbleibende Höhe vermessen wird. Dieses Ergebnis des Labortests wird anschließend mit dem Ergebnis des Testaufbaus in der Simulation verglichen und bei einer Abweichung der Ergebnisse über ein tolerierbares Maß mit abgeänderten Parametern wiederholt. Ein Vergleich der Ergebnisse aus dem Laborversuch mit der Simulation sind in Abb. 6 dargestellt.

Im zweiten Teil des Versuchs, ersichtlich in Abb. 5b, wird die Grundplatte, auf der der gesamte Versuch durchgeführt
Abb. 5: Schematische Darstellung des Prüfablaufs des Slump-Tests unterteilt in den ersten Schritt (a) und den zweiten Schritt (b) [6]
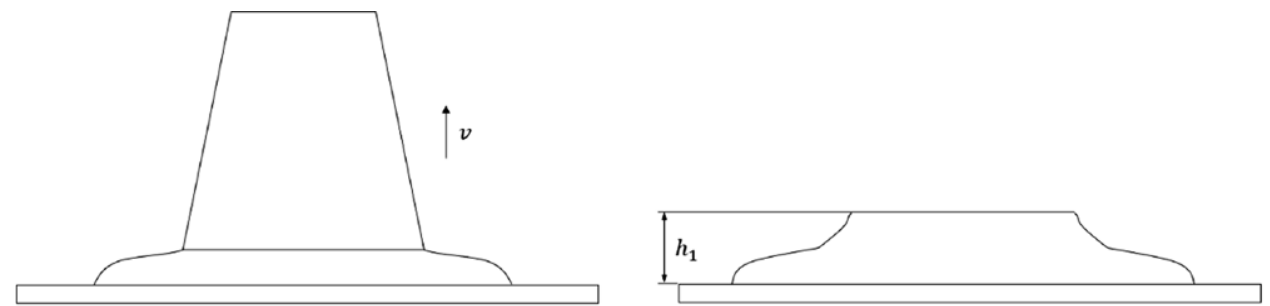

a
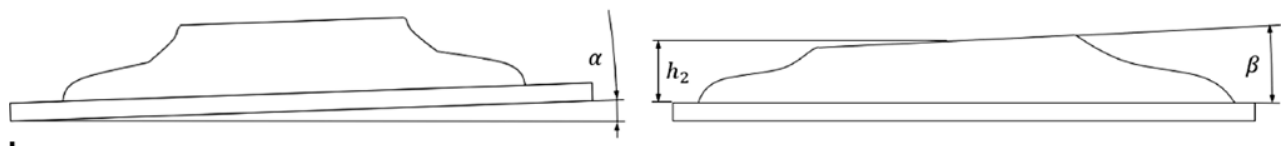

b
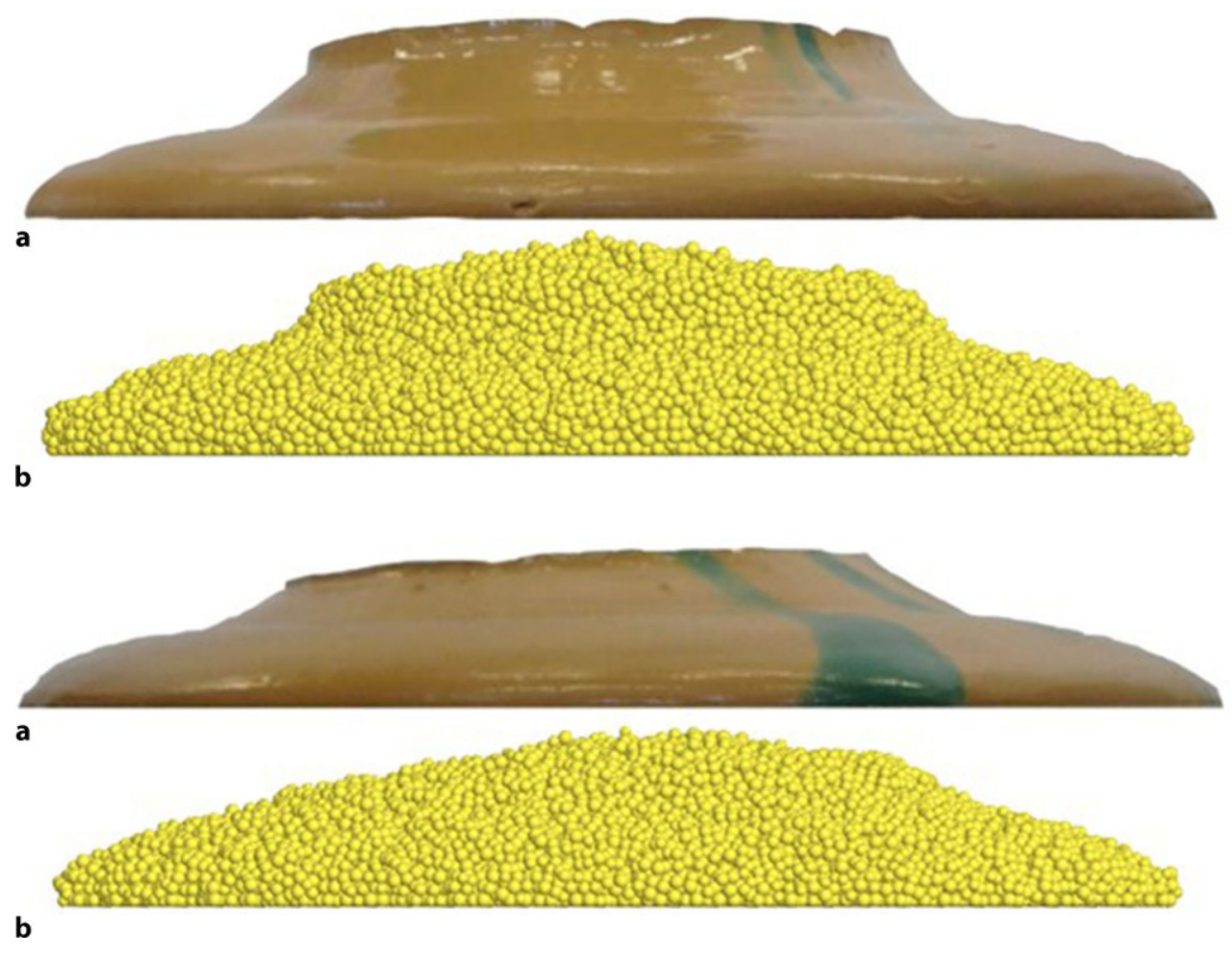

Abb. 6: Vergleich der Ergebnisse aus dem ersten Schritt des Versuchs (Abb. 5a), des Labortests (a) mit der DEM-Simulation (b) [6]

Abb. 7: Vergleich der Ergebnisse aus dem ersten Schritt des Versuchs (Abb. 5b), des Labortests (a) mit der DEM-Simulation (b) [6] 
wird, bis zum Anschlag angehoben und danach fallen gelassen.

Die nach DIN 12350-5 genormte Grundplatte ist mit Scharnieren ausgestattet und dadurch nur von einer Seite bis zu einem maximalen Winkel anhebbar, in Abb. 5b mit a bemaßt, was in einem asymmetrischen Einbruch des Materials durch den Aufprall resultiert, ersichtlich in Abb. 7. Dieser Schritt wird nur einmal und nicht, wie in der Norm vorgeschlagen, mehrmals durchgeführt, damit die Kontur des zusammengefallenen Materials intakt bleibt. Nachdem das Material nach dem Aufprall zur Ruhe gekommen ist, wird die verbleibende Höhe des abgesackten Materials und das asymmetrische Absacken des Plateaus, als $h$ und $b$ in Abb. 5b bemaßt, gemessen. Dabei wird das Aufprallverhalten des Materials kalibriert, was vor allem für eventuell folgende (fördertechnische) Simulationen in größerem Maßstab, z. B. Schurrensimulationen, interessant ist.

\section{Resümee}

Pastöse Materialien werden in den unterschiedlichsten technischen Verfahren eingesetzt, welche durch numerische Simulationen optimiert werden können. Das vorgestellte Kontaktmodell und die gezeigte Kalibrationsmethode bieten in diesem Zusammenhang die Möglichkeit, pastöses Materialverhalten mit diskreten Elementen abzubilden. Das Kontaktmodell basiert hierfür auf einem polynomischen Ansatz, und der für die Evaluierung dieses Modells vorgestellte Test wird auf einem standardisierter Slump-Test angewandt, um das charakteristische Verhalten pastöser Materialien widerzuspiegeln. Ein Abgleich zwischen Labor- und Simulationsmodell dient schlussendlich der Modellkalibration.

Finanzierung. Bereitstellung der Finanzierung des Open-Access durch die Montanuniversität Leoben. Die Recherche wurde im Rahmen eines Projektes, finanziert von FLSmidth USA, durchgeführt.

Interessenkonflikt. F. Platzer und E. Fimbinger geben an, dass kein Interessenkonflikt besteht.

Open Access Dieser Artikel wird unter der Creative Commons Namensnennung 4.0 International Lizenz veröffentlicht, welche die Nutzung, Vervielfältigung, Bearbeitung, Verbreitung und Wiedergabe in jeglichem Medium und Format erlaubt, sofern Sie den/die ursprünglichen Autor(en) und die Quelle ordnungsgemäß nennen, einen Link zur Creative
Commons Lizenz beifügen und angeben, ob Änderungen vorgenommen wurden.

Die in diesem Artikel enthaltenen Bilder und sonstiges Drittmaterial unterliegen ebenfalls der genannten Creative Commons Lizenz, sofern sich aus der Abbildungslegende nichts anderes ergibt. Sofern das betreffende Material nicht unter der genannten Creative Commons Lizenz steht und die betreffende Handlung nicht nach gesetzlichen Vorschriften erlaubt ist, ist für die oben aufgeführten Weiterverwendungen des Materials die Einwilligung des jeweiligen Rechteinhabers einzuholen.

Weitere Details zur Lizenz entnehmen Sie bitte der Lizenzinformation auf http://creativecommons.org/licenses/by/4.0/deed.de.

\section{Literatur}

1. Angelle Materials, Baton Rouge: Cement, concrete, slurry... what's the difference and which is best for my project? (2020). https:// www.angellematerials.com/blog/cement-concrete-slurrywhats-

the-difference-and-which-is-best-for-my-project. Zugegriffen: 20.12.2021.

2. Kralchevsky, P.: Capillary bridges and capillary-bridge forces, chapter 11. In: Kralchevsky, P.A., Nagayama, K. (Hrsg.) Particles at Fluid Interfaces and Membranes (Attachment of Colloid Particles and Proteins to Interfaces and Formation of Two-Dimensional Arrays), S. 469-502. Elsevier, Amsterdam (2001) https://doi.org/10.1016/S13837303(01)80052-1

3. Washino, K., Chan, E.L., Midou, H., Tsuji, T., Tanaka, T.: Tangential viscous force models for pendular liquid bridge of Newtonian fluid between moving particles. Chem. Eng. Sci. 174, 365-373 (2017). https://doi.org/10.1016/j.ces.2017.09.028

4. Wang, J.-P., Gallo, E., François, B., Gabrieli, F., Lambert, P.: Capillary force and rupture of funicular liquid bridges between three spherical bodies. Powder Technol. 305, 89-98 (2017). https://doi.org/10. 1016/j.powtec.2016.09.060

5. Liu, M.B., Liu, G.R.: Smoothed particle hydrodynamics (SPH): an overview and recent developments. Arch. Comput. Methods Eng. 17, 25-76 (2010). https://doi.org/10.1007/s11831-010-9040-7

6. Platzer, F., Fimbinger, E.: Modelling Pasty Material Behaviour Using the Discrete Element Method. Multiscale Science and Engineering. Springer, Luxemburg (2021) https://doi.org/10.1007/s42493-02100064-7

7. Gabrieli, F., Lambert, P., Cola, S., Calvetti, F.: Micromechanical modelling of erosion due to evaporation in a partially wet granular slope. Int. J. Numer. Anal. Meth. Geomech. (2011). https://doi.org/ 10.1002/nag. 1038

8. Hoornahad, H., Koenders, E.A.B.: Simulation of the Slump test based on the Discrete Element Method (DEM). Adv. Mater. Res. 446-449, 3766-3773 (2012). https://doi.org/10.4028/www.scientific. net/AMR.446-449.3766

Hinweis des Verlags. Der Verlag bleibt in Hinblick auf geografische Zuordnungen und Gebietsbezeichnungen in veröffentlichten Karten und Institutsadressen neutral. 